\title{
Surgeons blinded by enhanced navigation: the effect of augmented reality on attention.
}

Benjamin J. Dixon MBBS ${ }^{1,2}$, Michael J. Daly, $\mathrm{MSc}^{3,4}$, Harley Chan, $\mathrm{PhD}^{3}$, Allan D Vescan, $\mathrm{MD}^{5}$, Ian J. Witterick, $\mathrm{MD}^{1,2,5}$, Jonathan C. Irish, $\mathrm{MD}^{1,2}$

${ }^{1}$ Departments of Surgical Oncology, ${ }^{2}$ Otolaryngology-Head and Neck Surgery, ${ }^{3}$ Ontario Cancer Institute, ${ }^{4}$ Institute of Medical Science, Princess Margaret Hospital, University Health Network, University of Toronto, Toronto, Ontario, Canada

Department of ${ }^{5}$ Otolaryngology-Head and Neck Surgery, Mount Sinai Hospital, University of Toronto, Toronto, Ontario, Canada

Financial disclosures: Wet lab instruments and devices supplied by Karl Storz Endoscopy Canada and Medtronic of Canada.

Conflict of interest: Wet lab instruments and devices supplied by Karl Storz Endoscopy Canada and Medtronic of Canada.

Keywords: Augmented reality, inattentional blindness, attentional tunneling, image guided surgery, endoscopic surgery, surgical navigation

Original article

Send Correspondence to:

Jonathan Irish, MD

610 University Avenue, 3-954

Toronto, ON M5G 2M9, Canada

E-mail: jonathan.irish@uhn.on.ca 


\begin{abstract}
Background: Advanced image-guidance systems, allowing presentation of 3-dimensional navigational data in real-time, are being enthusiastically developed for many medical procedures. Other industries, including aviation and military, have noted that shifting attention towards such compelling assistance has detrimental effects. Through the detection rate of unexpected findings, we assess whether inattentional blindness is significant in a surgical context and evaluate the impact of on-screen navigational cuing with augmented reality.
\end{abstract}

Methods: Surgeons and trainees performed an endoscopic navigation exercise on a cadaveric specimen. Subjects were randomised to either a standard endoscopic view (control) or an augmented reality (AR) view consisting of endoscopic video fused with anatomical contours. Two unexpected findings were presented in close proximity to the target point; one critical complication and one foreign body (screw). Task completion time, accuracy and recognition of findings were recorded.

Results: Detection of the complication was $0 / 15$ in the AR group versus $7 / 17$ in the control $(\mathrm{p}=0.008)$. Detection of the screw was $1 / 15(\mathrm{AR})$ and $7 / 17$ (control) $(\mathrm{p}=0.041)$. Recognition of either finding was $12 / 17$ and $1 / 15$ for the control and AR group respectively $(\mathrm{p}<0.001)$. Accuracy was greater for the AR group versus control group with median (IQR) distance from the target point measuring 2.10mm (1.29-2.37) Vs 4.13 (3.11-7.39) respectively $(\mathrm{p}<0.001)$. 
Conclusion: Inattentional blindness was evident in both groups. Although more accurate, the AR group were less likely to identify significant unexpected findings clearly within view. Advanced navigational displays may increase precision but strategies to mitigate attentional costs need further investigation to allow safe implementation.

\section{Introduction}

There has been an increasing trend towards endoscopic, image-guided and minimally invasive techniques in most procedural specialties. In comparison to open approaches, minimally invasive techniques may provide a narrower field of view and fewer spatial cues. This has resulted in a greater reliance on technology to aid in diagnosis and precise navigation. As these procedures have evolved there have been parallel advances in imaging technology which can provide an accurate sub-millimetre, 3-dimensional (3-D) representation of the operative field. Not surprisingly, there have been ever increasing efforts to utilise this data in a more dynamic way while performing tasks.

Image-guided surgery (IGS) systems are now commonly used for many procedures, ${ }^{1}$ especially when the operative site is within a relatively rigid framework such as neurosurgery, skull base and sinonasal surgery. In such areas, IGS systems have been shown to significantly change surgical strategy. ${ }^{2}$ Technical advancements in intraoperative imaging and deformable registration ${ }^{3}$ are now allowing IGS to be used in areas where soft-tissue deformation and artefact from respiratory movement and arterial pulsation had previously deemed this technology unviable. ${ }^{4,5}$ The Guided Therapeutics program at University Health Network, Toronto, has developed a navigation system which incorporates intraoperative imaging and image registration. ${ }^{6,7}$ Anatomical 
segmentation and endoscope tracking then allows 3-D representation of imaging and contouring data which can be used during endoscopic surgery in real-time. ${ }^{8}$ This can be displayed as a virtual view on a sub-monitor or fused with the main endoscopic image as augmented reality. See Figure 1.

Augmented reality (AR) describes the placement of virtual objects within a real environment in real-time, meaning true and virtual images are combined in a dynamic fashion. ${ }^{9}$ This technique holds the promise of displaying accurate anatomical data, derived from adjunct imaging, on the endoscopic monitor during a procedure. Intuitively most physicians recognise the potential clutter or visual overstimulation inherent in such a display. While many research groups, including ours, have reported on the potential clinical benefits of this technology ${ }^{8,10-12}$, the possible detrimental effects of augmented reality displays remain poorly investigated or discussed.

Human factors and psychological studies suggest that compelling displays like AR alter attentional focus while performing tasks. Even in the absence of additional cues, when people concentrate on a task they may fail to recognise highly notable events or objects that appear within their visual field. This phenomenon, known as inattentional blindness, becomes more evident when tasks demand greater attention. ${ }^{13}$ One might expect that accurate and accessible navigational data would decrease cognitive demand during a procedure and allow for a greater allocation of attention to alternative tasks ${ }^{14}$, however, evidence from the aviation, military and auto industries suggests that the opposite occurs. Drawing attention to a certain cue in fact increases the chance of missing unexpected but important events occurring in the same environment. ${ }^{15,16}$ 
In order to refine the user interface of our image-guidance system, we aimed to assess the impact of augmented reality on visual attention using a randomised-controlled trial comparing conventional endoscopy to an AR view. See Figure 1a\&b. Endoscopic transnasal skull base navigation in a cadaveric specimen was chosen as the surgical model as it provides a complex 3-D environment within a rigid framework that can be accurately registered. Endoscopic approaches to skull base tumors are increasingly popular and the use of image guidance in this setting is almost ubiquitous. The main outcome measure was the detection rate of two, deliberately presented, unexpected findings: one critical complication and one foreign body. Previous studies investigating inattentional blindness presented findings that were clearly out of place and the foreign body (a screw) was chosen to replicate these experiments in a surgical setting. We also wished to present a surgically relevant abnormality so the critical complication (injured optic nerve) was added. Importantly, we did not aim to assess the validity of any surgical approach or technique, nor the competence of the participants. Rather we wished to assess the effect on-screen cuing with AR had on detection of unexpected findings as a measure of the potential attentional cost of these systems.

\section{Methods}

Otolaryngology trainees, fellows and staff surgeons participated in the trial on a fully functional, dynamic endoscopic AR prototype after informed consent and institutional ethics approval. A single cadaver head underwent a computed tomography (CT) scan after fixation of fiduciary markers. Anatomical contours of critical structures were then manually delineated using ITK-Snap (University of Pennsylvania, PA). ${ }^{17}$ Figure 1c. 
Sinonasal dissection, including ethmoidectomy and a wide unified sphenoidotomy, was then carried out consistent with exposure for trans-sphenoidal skull base surgery such as pituitary surgery. A post-ablation cone-beam CT image was acquired and contours were rigidly registered to this scan. ${ }^{6}$

An optical IGS (Polaris, NDI, Waterloo, Ontario) system was used with custom visualization software for real-time tracking of a 0 -degree endoscope (Hopkins II telescope and IMAGE1 camera, Karl Storz, Tuttlingen, Germany) and a probe. A dynamic virtual endoscopic image could then be displayed by registering imaging and contouring data to the head. ${ }^{8}$

Two unexpected findings were present in the specimen. Firstly, a foreign object (small screw) was placed within the sphenoid sinus. Secondly, the optic nerve was divided at the chiasm, dissected along the canal and draped into the sinus. This represented one totally unexpected finding and one critical complication. See Figure 1(a). Subjects were then randomised to perform a navigation task in one of two endoscopic environments:

A. Standard Endoscopy (Control group)- The image was adjusted to $75 \%$ opacity to match the image quality of the augmented reality view but no contour fusion was added. Figure 1(a)

B. Augmented reality (AR group)- The endoscopic view was adjusted to $75 \%$ opacity with anatomic contours seen as a fusion in the remaining $25 \%$. Figure 1(b) 
Each subject undertook the following task after randomization to one arm. A briefing outlined the prior dissection, the AR technology and the anatomical contours. Unaware as to which view they would receive, each participant was asked to localize, with a tracked pointer, the anterior face of the sella turcica (pituitary fossa) in the midline as accurately and efficiently as possible. The AR group viewed pre-contoured anatomical data fused with the endoscopic image which updated dynamically, in real-time, as the subject moved the endoscope. Subjects were aware they were being timed but no time limits were enforced. Randomization was stratified to balance the level of surgical experience in each group.

Once the subject had indicated they had localised the target area the 3-D coordinates and time were recorded and the endoscopic image was hidden. The following questions were then asked: 1) Did you notice anything unusual? 2) Did you note any critical complication? 3) Did you notice a foreign body in the sphenoid sinus? 4) Did you notice a screw? Binary responses (yes/no) were documented. Video footage of the monitor and room were separately recorded.

\section{Statistical analysis}

Analysis was performed using SPSS Statistics 19 (Chicago, Illinois, USA). Recognition rate of unexpected findings between the two groups was compared using two-sided Fisher's exact test. Spearman's rank correlations were performed to identify any significant factors associated with recognition of the findings. Binary logistic regression analysis, with Wald forward and backward modeling, was performed to assess whether randomization group, time taken or experience level were independently associated with 
noting an unexpected finding. Task accuracy was assessed by measuring distance from a "gold standard" target point set by the investigators aided by image guidance. Linear regression analysis was used to again assess the impact of group, experience level and time on accuracy.

\section{Results}

A total of 33 otolaryngology residents, fellows and surgeons spanning all levels of experience were enrolled in the study. There was an equal distribution of experience levels between groups (likelihood ratio 0.98). One subject in the AR group was excluded from analysis as the AR malfunctioned due to processing latency in the laptop computer, freezing the superimposed virtual view in a static position. Retrospective scrutiny of individual video footage revealed that both unexpected findings were clearly seen on the monitor in every instance.

\section{Recognition of unexpected findings}

Of the 32 subjects included, only four noticed something unusual (question 1) with two commenting during the exercise.

Direct questioning regarding noticing a critical complication (question 2) revealed correct answers in 7 of $17(41 \%)$ in the control group and 0 of $15(0 \%)$ in the AR group $(\mathrm{p}=0.008)$. The same ratios were present for question 3 regarding noticing a foreign body.

See Table 1. One subject from the AR group altered their answer for question 4 (did you see a screw?) from their response to question 3, meaning 1 of 15 (7\%) in the AR group noticed a screw compared to 7 of 17 in the control group $(\mathrm{p}=0.041)$. In total 12 (5 
complication, 5 foreign body, 2 both) noticed a finding in the control group compared to one in the AR group $(\mathrm{p}<0.001)$.

There was a trend towards shorter task completion time for the AR group $(\mathrm{p}=0.153)$.

Table 1. Logistic regression analysis revealed that randomization group was the only independent significant variable for all unexpected findings (all $p<0.03)$. After controlling for display group, time and experience level were not significant.

Median (inter-quartile range (IQR)) task completion time for the sub-group reporting a finding (28 seconds (22.5-33)) was significantly longer than for those who missed the findings $(19(12-30))(\mathrm{p}=0.03$ Mann Whitney $U$ test $)$. There was a trend towards faster completion time in the more experienced subjects $(\mathrm{R}=0.33 \mathrm{p}=0.064$ Spearman's correlation coefficient).

\section{Accuracy}

A 3-D representation of the localised points with target reference is shown in Figure 2(a). Median (IQR) distance from the designated target point was significantly smaller in the AR group (2.10mm (1.29-2.37)) than the control group (4.13mm $(3.11-7.39))(\mathrm{p}<0.001)$. Table 1. Display type was the only significant variable for accuracy, however, there was a trend towards greater accuracy with experience $(p=0.074)$. Localised points are graphically represented on a CT image as an anatomical reference in Figure 2(b).

\section{Discussion}


This study provides experimental evidence that surgeons exhibit inattentional blindness while performing tasks. We have also demonstrated that recognition of significant, unexpected findings can be markedly reduced when augmented reality is used to enhance navigation. Despite the expansion of minimally invasive techniques, image-guided procedures and telerobotic surgery, the potential detrimental effects of advanced displays, including augmented reality, are poorly explored in the medical literature. There have been some studies in anaesthesia looking at the pros and cons of head mounted AR displays for monitoring purposes but investigation in other fields is sparse. ${ }^{18,19}$ Although perceptual issues with medical AR are well documented ${ }^{20}$ we believe this is the first experiment to demonstrate that augmented surgical navigation systems have a potentially deleterious attentional cost. This has implications on the use and development of all image-guided procedures.

In our study, on-screen navigation improved accuracy and there was a trend towards faster task completion. A trial investigating navigation on an airport surface in reduced visibility showed similar results. The addition of an augmented display resulted in more accurate navigation, faster route completion and decreased pilot task workloads. ${ }^{21}$ Emulating such advances in a medical context is clearly appealing; a safer, more efficient procedure, with less demand on the physician. Our data supports other authors who suggest that such gains may be achievable for medical procedures, ${ }^{11,22,23}$ but safe implementation will require some mitigation of attentional costs.

The presence of inattentional blindness (IB) while performing a surgical task, was expected, given results from similar studies investigating visual perception. Even so, 
when designing this experiment it seemed inconceivable that surgeons would miss the findings that were so clearly visible within the operative field. One famous example of IB is colloquially termed the "invisible gorilla", where a person in a gorilla costume is clearly present on a monitor that the subjects were viewing while focused on a task. ${ }^{13}$ Amazingly during one condition, 58\% of subjects missed the gorilla. Similarly, 59\% of our "task focused" control arm failed to notice the screw even though it would have been difficult to place it any closer to the target point. Figure 1(a).

Perhaps of greater significance is the fact that no one in the AR group noted the complication and only one saw the screw. Military and aviation studies have previously documented similar drawbacks when investigating AR and head-up displays. The term "attentional tunneling" is used to describe cognitive fixation on specific cues and the resulting cost of neglecting alternative information or tasks. ${ }^{24}$ This phenomenon has been implicated in the Three Mile Island nuclear accident ${ }^{24}$, F16 mishaps in the US Air Force ${ }^{25}$ and cell phone related car accidents. ${ }^{26,27}$ In a military simulation there was reduced detection of unexpected but "highest priority" objects when closely presented adjacent to cued (with AR) targets. ${ }^{15}$ The participant's attention was therefore drawn to the highlighted object even though the non-cued item nearby was of greater importance. Attentional constraints have been shown to severely limit a pilot's ability to simultaneously process real world and augmented display information. ${ }^{28}$ Head-up display symbology may support precision landing, however, attraction to the displayed image has led attention away from runway incursions ${ }^{29}$ and has resulted in reduced detection of flight path objects as salient as a blimp. ${ }^{30}$ 
As we did not analyse eye movements we are unable to determine whether failure to note the unexpected objects was due to failure to scan the area or failure to perceive. Radiological studies suggest both occur and explain the "satisfaction of search" phenomenon whereby secondary abnormalities are missed on imaging studies. Greater attention, measured by gaze dwell time, is given to contrast enhanced regions of a radiograph compared to non-contrast regions, leading to missed abnormalities. ${ }^{31}$ This differs from other studies of eye-position during radiograph interpretation which indicated that the effect is based on failure to recognise. ${ }^{32}$ The addition of computer-aided diagnosis in medical imaging has been shown to reduce the visual search for non-cued abnormalities. ${ }^{33}$ The differences between our randomization groups suggest that AR has a similar effect to contrast on an imaging study; secondary findings are overlooked due to an attentional shift towards a highlighted area.

In addition to attentional tunneling, the clutter caused by on-screen cues provides another explanation for the inferior detection of unexpected findings. As more and more information is displayed within one's visual field the costs of clutter begin to outweigh the costs of scanning. ${ }^{34,35}$ In a procedural context this suggests that there becomes a point where viewing imaging data away from the operative field in a traditional manner may be safer and faster than interpreting information rich data within the field, despite the inherent shift of attention. Clutter and jitter induced by AR may reduce the user's effective visual acuity. ${ }^{36}$ We believe there may be an additional camouflage effect as evidenced by the difficulty in seeing the optic nerve when fused with the red carotid artery contour. Figure 1(b). 
Given our findings, we would caution any claims made in the medical literature that advanced navigational displays will inevitably lead to safer, easier and more precise surgery. Technical development in many disciplines has been impressive, including the introduction of live AR through continuous low dose CT scanning, AR assistance for remote neurosurgical assistance, fused video-ultrasound images for partial nephrectomy, AR assisted navigation during natural orifice translumenal endoscopic surgery (NOTES) and overlay AR during robotic surgery., ${ }^{4,11,12,37}$ Reporting of this progress remains focused on registration accuracy, and perhaps rightly so given it is absolutely essential for reliable navigation. However, registration error represents only one of many potential detrimental effects of AR, which include attention shifts, the behavioural effects of reliance, complacency, trust, the impact of automation failure and the processing of noncued targets and cued non-targets. ${ }^{15,16,38}$ In light of these other potential hazards, further investigation into interface design is required.

Although the sample size is relatively small it would be challenging to find a greater number of surgeons with varying levels of experience to participate and perform such specific and surgically relevant tasks in a realistic environment. Similar human factors studies investigating skilled end-user's use of technology have been equally limited in subjects. ${ }^{16,30,34}$ A single cadaveric specimen was used. Although individual anatomic variations exist we doubt this would significantly affect the results. We believe the greatest factor limiting interpretation of these results is that none of the participants had experience with operative AR. We are unsure whether lack of familiarity affected outcome. Perhaps the difference between the groups would have been insignificant if they had time to use the system and get used to the display. On-screen AR could 
potentially be presented in various formats and we investigated only one setting. It could be argued that an alternative view, such as a wire mesh overlay rather than solid contours or a reduced contour opacity, could limit or negate the effect demonstrated. The unknown effect of habituation to the system (learning curve) and the possibility of alternative views, highlights the need for further research into display settings and feedback mechanisms. Despite these limitations, our findings correlate closely with results in other industries thus we feel the effect demonstrated does exist. Potential methods to reduce inattentional blindness that require further investigation include on-demand rather than full-time AR, clutter reduction, sonifications (informative auditory feedback) ${ }^{39}$ or a perceptually matched, 3-D virtual view on a sub-monitor.

Although the task chosen for the trial was designed to focus attention, it is important to remember that the only parameter that differed between the groups was the display setting. The on-screen cuing appears to have a far greater impact on detection of unexpected findings than either experience level or time spent on the task. Despite the attentional costs, we see enormous potential benefits of real-time navigation and we are actively engaged in addressing the issues of attention and distraction. Our attentional capacity is limited and we focus it for good reason; many tasks require concentration and unexpected events are rare. Thus quarantining a portion of our attention for unexpected events is wasteful in most circumstances and probably impossible anyway. Instead we suggest that surgeons need to be aware of the potential detrimental effects of additional stimuli while performing procedures as advanced displays continue to be adopted into practice. Procedures and sub-tasks with high navigational demand should be targeted and systems should be customised to mitigate distractive cues when demand is low. Emphasis 
needs to be placed on the perceptual limitations of the task focused end-user therefore it is essential that physicians play a role in the development of these systems.

Figure Legends

Figure 1. (a) A still screen-shot taken during the endoscopic trial of the standard view with opacity at $75 \%$. Relevant findings labeled including foreign body and complication. (b) Same image as (a) with AR fusion at $25 \%$. This is an actual screen-shot from a participant in the AR group who missed both findings. (c) Example of virtual view of contours with wire mesh surface rendering. (d) Trial set up displaying monitor and optical IGS.

Figure 2. (a) 3-D virtual view displaying the points localised by the control group (magenta) and the AR group (blue). The reference target point is in yellow (partially obscured). The AR group was more accurate $(\mathrm{p}<0.001)$. (b) The 3-D data points from (a) are shown projected onto a sagittal CT image for anatomical reference.

Financial disclosures: Wet lab instruments and devices were on loan from Karl Storz Endoscopy Canada and Medtronic of Canada. This work is supported by the Guided Therapeutics (GTx) Program at the University Health Network, including The Kevin and Sandra Sullivan Chair in Surgical Oncology, The Hatch Engineering Fellowship Fund, The RACH Fund, and the Princess Margaret Hospital Foundation. None of the authors have any corporate or commercial relationships.

\section{Acknowledgements}

Thank you to Karl Storz Endoscopy Canada and Medtronic of Canada for providing wet lab dissection instruments and devices. The prototype cone-beam CT C-arm was developed in collaboration with Siemens Healthcare. This study could not have been performed without assistance from staff at the University of Toronto Surgical Skills

Centre, Mount Sinai Hospital, Toronto. This work is supported by the Guided Therapeutics (GTx) Program at the University Health Network, including The Kevin and 
Sandra Sullivan Chair in Surgical Oncology, The Hatch Engineering Fellowship Fund,

The RACH Fund, and the Princess Margaret Hospital Foundation.

\section{References}

1. Cleary K, Peters TM. Image-guided interventions: technology review and clinical applications. Annu Rev Biomed Eng;12: 119-142.

2. Strauss G, Koulechov K, Rottger S, Bahner J, Trantakis C, Hofer M, Korb W, Burgert O, Meixensberger J, Manzey D, Dietz A, Luth T. Evaluation of a navigation system for ENT with surgical efficiency criteria. Laryngoscope 2006;116(4): 564-572.

3. Nithiananthan S, Brock KK, Daly MJ, Chan H, Irish JC, Siewerdsen JH. Demons deformable registration for CBCT-guided procedures in the head and neck: convergence and accuracy. Med Phys 2009;36(10): 4755-4764.

4. Shekhar R, Dandekar O, Bhat V, Philip M, Lei P, Godinez C, Sutton E, George I, Kavic S, Mezrich R, Park A. Live augmented reality: a new visualization method for laparoscopic surgery using continuous volumetric computed tomography. Surg Endosc;24(8): 1976-1985.

5. Cheung CL, Wedlake C, Moore J, Pautler SE, Peters TM. Fused video and ultrasound images for minimally invasive partial nephrectomy: a phantom study. Med Image Comput Comput Assist Interv; 13(Pt 3): 408-415.

6. Siewerdsen JH, Daly MJ, Chan H, Nithiananthan S, Hamming N, Brock KK, Irish JC. High-performance intraoperative cone-beam CT on a mobile C-arm: an integrated system for guidance of head and neck surgery. Proc SPIE 2009;7261(1): 72610J.

7. Daly MJ, Chan H, Nithiananthan S, Qiu J, Barker E, Bachar G, Dixon BJ, Irish JC, Siewerdsen JH. Clinical implementation of intraoperative cone-beam CT in head and neck surgery. In: Kenneth HW, David RH, III, editors.: SPIE. p. 796426.

8. Daly MJ, Chan H, Prisman E, Vescan A, Nithiananthan S, Qiu J, Weersink R, Irish JC, Siewerdsen JH. Fusion of intraoperative cone-beam CT and endoscopic video for image-guided procedures. Proc SPIE 2010;7625(1): 762503.

9. Azuma RT. A Survey of Augmented Reality. In Presence: Teleoperators and Virtual Environments 1997; 4: 355-385.

10. Teber D, Guven S, Simpfendorfer T, Baumhauer M, Guven EO, Yencilek F, Gozen AS, Rassweiler J. Augmented reality: a new tool to improve surgical accuracy during laparoscopic partial nephrectomy? Preliminary in vitro and in vivo results. Eur Urol 2009;56(2): 332-338.

11. Volonte F, Pugin F, Bucher P, Sugimoto M, Ratib O, Morel P. Augmented reality and image overlay navigation with OsiriX in laparoscopic and robotic surgery: not only a matter of fashion. J Hepatobiliary Pancreat Sci 2011.

12. Fernandez-Esparrach G, San Jose Estepar R, Guarner-Argente C, Martinez-Palli G, Navarro R, Rodriguez de Miguel C, Cordova H, Thompson CC, Lacy AM, Donoso L, Ayuso-Colella JR, Gines A, Pellise M, Llach J, Vosburgh KG. The role of a computed tomography-based image registered navigation system for natural orifice transluminal endoscopic surgery: a comparative study in a porcine model. Endoscopy;42(12): 10961103. 
13. Simons DJ, Chabris CF. Gorillas in our midst: sustained inattentional blindness for dynamic events. Perception 1999;28(9): 1059-1074.

14. Carswell CM, Clarke D, Seales WB. Assessing mental workload during laparoscopic surgery. Surg Innov 2005;12(1): 80-90.

15. Yeh M, Wickens CD. Display signaling in augmented reality: effects of cue reliability and image realism on attention allocation and trust calibration. Hum Factors 2001;43(3): 355-365.

16. Maltz M, Shinar D. New alternative methods of analyzing human behavior in cued target acquisition. Hum Factors 2003;45(2): 281-295.

17. Yushkevich PA, Piven J, Hazlett HC, Smith RG, Ho S, Gee JC, Gerig G. Userguided 3D active contour segmentation of anatomical structures: significantly improved efficiency and reliability. Neuroimage 2006;31(3): 1116-1128.

18. Liu D, Jenkins SA, Sanderson PM. Patient monitoring with head-mounted displays. Curr Opin Anaesthesiol 2009;22(6): 796-803.

19. Liu D, Jenkins SA, Sanderson PM, Fabian P, Russell WJ. Monitoring with headmounted displays in general anesthesia: a clinical evaluation in the operating room. Anesth Analg;110(4): 1032-1038.

20. Sielhorst T, Feuerstein, M., Navab, N. Advanced Medical Displays: A Literature Review of Augmented Reality. Journal of Display Technology 2008;VOL. 4, (NO. 4, DECEMBER 2008): 451-467.

21. McCann RS, Foyle, D.C., Andre, A.D., Battiste, V. Advanced navigation aids in the flight deck: Effects on ground taxi performance under low visibilty conditions. SAE Transactions: Journal of Aerospace 1996;105: 1419-1430.

22. Marescaux J, Rubino F, Arenas M, Mutter D, Soler L. Augmented-reality-assisted laparoscopic adrenalectomy. JAMA 2004;292(18): 2214-2215.

23. Kawamata T, Iseki H, Shibasaki T, Hori T. Endoscopic augmented reality navigation system for endonasal transsphenoidal surgery to treat pituitary tumors: technical note. Neurosurgery 2002;50(6): 1393-1397.

24. Wickens CD. Attentional tunneling and task management: Technical Report AHFD-05-23/NASA-05-10. Urbana-Champaign, IL: University of Illinois; 2005.

25. Shappell SA, Wiegmann, D.A. A human error analysis of general aviation controlled flight into terrain accidents occurring between 1990-1998 (Final Rep. DOT/FAA/AM-03/4). Washington, DC: Office of Aerospace Medicine; 2003.

26. Strayer DL, Johnston, W.A. Driven to distraction: Duel-task studies of simulated driving and conversing on cellular telephone. Psychological Science 2001;12(6): 462466.

27. Horrey WJ, Wickens CD. Examining the impact of cell phone conversations on driving using meta-analytic techniques. Hum Factors 2006;48(1): 196-205.

28. McCann RS, Foyle, D.C., Johnston, J.C. Attentional limitations with head-up displays. In: Jensen RS, editor. Proceedings of the Seventh International Symposium on Aviation Psychology 1993; Colombus, OH; 1993. p. 70-75.

29. Fischer E, Haines, R.F., Price, T.A. Cognitive issues in head-up displays (NASA Technical paper 1711). Moffat Field, CA: NASA Ames Research Center; 1980.

30. Wickens CD, Alexander, A.L., Horrey, W.J., Nunes, A., Hardy, T.J. Traffic and flight guidance depiction on a synthetic vision system display: The affects of clutter on performance and visual attention allocation. In: Proceedings of the 48th Annual Meeting 
of the Human Factors and Ergonomics Society; 2004; Santa Monica, CA: HFES; 2004. p. 218-222.

31. Berbaum KS, Franken EA, Jr., Dorfman DD, Miller EM, Krupinski EA, Kreinbring K, Caldwell RT, Lu CH. Cause of satisfaction of search effects in contrast studies of the abdomen. Acad Radiol 1996;3(10): 815-826.

32. Samuel S, Kundel HL, Nodine CF, Toto LC. Mechanism of satisfaction of search: eye position recordings in the reading of chest radiographs. Radiology 1995;194(3): 895902.

33. Berbaum KS, Caldwell RT, Schartz KM, Thompson BH, Franken EA, Jr. Does computer-aided diagnosis for lung tumors change satisfaction of search in chest radiography? Acad Radiol 2007;14(9): 1069-1076.

34. Yeh M, Merlo JL, Wickens CD, Brandenburg DL. Head up versus head down: the costs of imprecision, unreliability, and visual clutter on cue effectiveness for display signaling. Hum Factors 2003;45(3): 390-407.

35. Ververs PM, Wickens CD. Head-up displays: effects of clutter, display intensity, and display location on pilot performance. Int J Aviat Psychol 1998;8(4): 377-403.

36. Livingston MA. Evaluating human factors in augmented reality systems. IEEE

Comput Graph Appl 2005;25(6): 6-9.

37. Shenai MB, Dillavou M, Shum C, Ross D, Tubbs RS, Shih A, Guthrie BL.

Virtual interactive presence and augmented reality (VIPAR) for remote surgical assistance. Neurosurgery;68(1 Suppl Operative): 200-207; discussion 207.

38. Lee JD, See KA. Trust in automation: designing for appropriate reliance. Hum Factors 2004;46(1): 50-80.

39. Sanderson PM, Liu D, Jenkins SA. Auditory displays in anesthesiology. Curr Opin Anaesthesiol 2009;22(6): 788-795.

Table 1.

\begin{tabular}{|c|c|c|c|c|}
\hline & & $\begin{array}{c}\text { Group A } \\
\text { Standard endoscopy }\end{array}$ & $\begin{array}{c}\text { Group B } \\
\text { Augmented reality }\end{array}$ & Significance \\
\hline \multirow[t]{4}{*}{ Subjects } & Total $n=32$ & 17 & 15 & \multirow{4}{*}{$\begin{array}{l}\mathrm{p}=0.98 \\
\text { Likelihood ratio }\end{array}$} \\
\hline & PGY $1,2 n=8$ & 4 & 4 & \\
\hline & PGY 3-5 n=13 & 7 & 6 & \\
\hline & $\begin{array}{l}\text { Fellows/ surgeons } \\
\mathrm{n}=11\end{array}$ & 6 & 5 & \\
\hline $\begin{array}{l}\text { Accuracy (mm } \\
\text { from target) }\end{array}$ & Median (IQR) & $4.13(3.11-7.39)$ & $2.10(1.29-2.37)$ & $\begin{array}{l}\mathrm{P}<0.001 \text { Mann- } \\
\text { Whitney U test }\end{array}$ \\
\hline
\end{tabular}




\begin{tabular}{|c|c|c|c|c|}
\hline \multirow{2}{*}{$\begin{array}{c}\text { Complication } \\
\text { noted }\end{array}$} & YES & 7 & 0 & \multirow{2}{*}{$\begin{array}{l}\mathrm{p}=0.008 \text { Fisher's } \\
\text { exact test }\end{array}$} \\
\hline & $\mathrm{NO}$ & 10 & 15 & \\
\hline \multirow{2}{*}{$\begin{array}{c}\text { Foreign body } \\
\text { noted }\end{array}$} & YES & 7 & 0 & \multirow{2}{*}{$\begin{array}{l}\mathrm{p}=0.008 \text { Fisher's } \\
\text { exact test }\end{array}$} \\
\hline & $\mathrm{NO}$ & 10 & 15 & \\
\hline \multirow[t]{2}{*}{ Screw noted } & YES & 7 & 1 & \multirow{2}{*}{$\begin{array}{l}p=0.04 \text { Fisher's } \\
\text { exact test }\end{array}$} \\
\hline & $\mathrm{NO}$ & 10 & 14 & \\
\hline \multirow{2}{*}{$\begin{array}{c}\text { Either finding } \\
\text { noted }\end{array}$} & YES & 12 & 1 & \multirow{2}{*}{$\begin{array}{l}\mathrm{p}<0.001 \text { Fisher's } \\
\text { exact test }\end{array}$} \\
\hline & $\mathrm{NO}$ & 5 & 14 & \\
\hline $\begin{array}{c}\text { Time to } \\
\text { complete task }\end{array}$ & $\begin{array}{c}\text { Median (IQR) } \\
\text { seconds }\end{array}$ & $25(21-30)$ & $19(12-31.5)$ & $\begin{array}{l}\mathrm{p}=0.15 \text { Mann- } \\
\text { Whitney U test }\end{array}$ \\
\hline
\end{tabular}

Table1. Difference in accuracy, detection of unexpected findings and task completion time between the randomisation groups. 


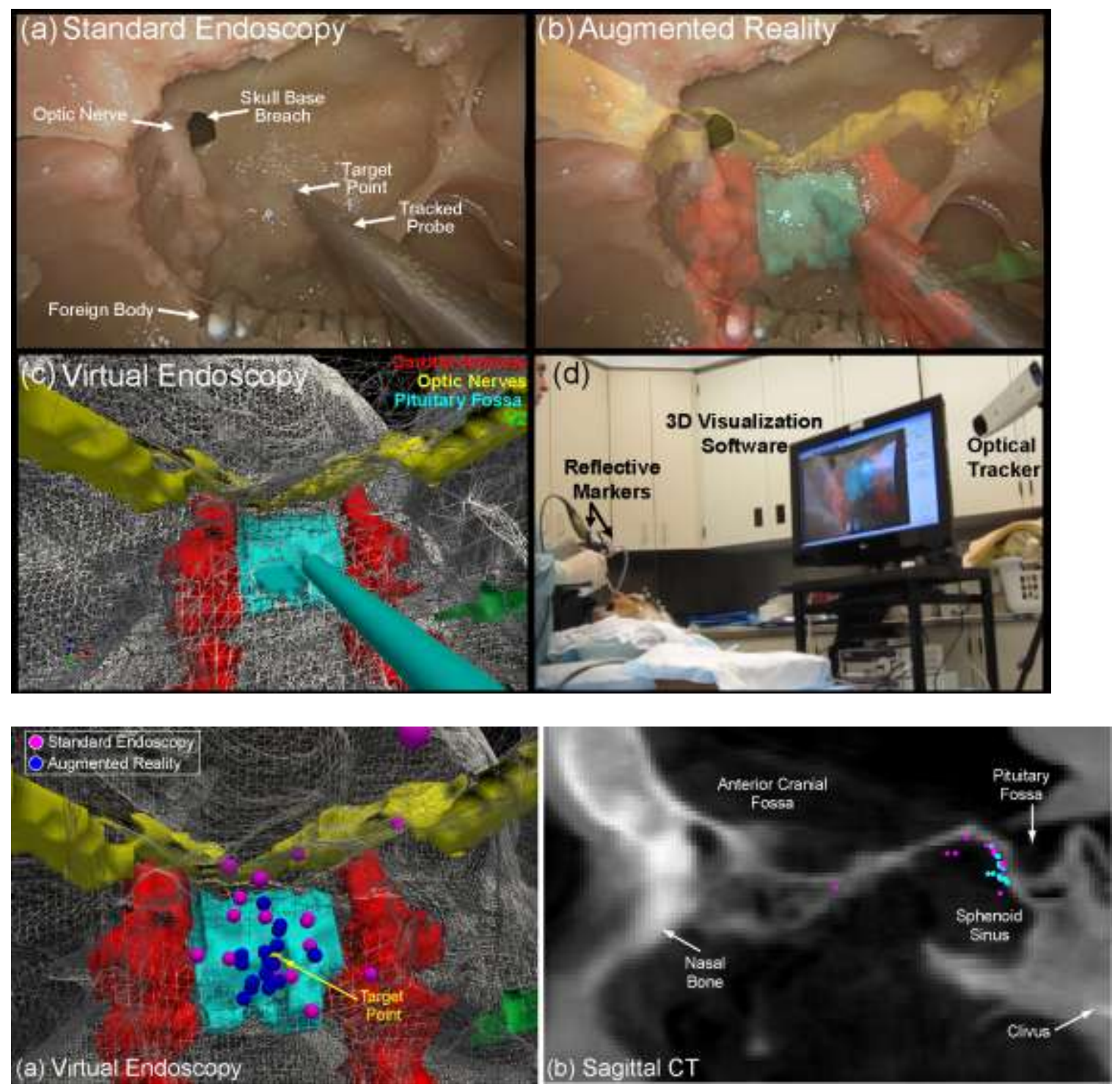

